2. Jesse Douglas, Solution of the problem of Plateau, Trans. Amer. Math. Soc. vol. 33 (1931) pp. 263-321.

3. J. Hadamard, Sur le principe de Dirichlet, Bull. Soc. Math. France vol. 34 (1906) pp. 135-138.

4. Oliver Dimon Kellogg, Foundations of potential theory, Berlin, Springer, 1929.

Alabama Polytechnic Institute

\title{
NON-MEASURABLE SETS AND THE EQUATION
}

$$
\begin{gathered}
f(x+y)=f(x)+f(y) \\
\text { ISRAEL HALPERIN }
\end{gathered}
$$

1. A set of $S$ real numbers which has inner measure $m_{*}(S)$ different from its outer measure $m^{*}(S)$ is non-measurable. An extreme form, which we shall call saturated non-measurability, occurs when $m_{*}(S)=0$ but $m^{*}(S M)=m(M)$ for every measurable set $M, m(M)$ denoting the measure of $M$. This is equivalent to: both $S$ and its complement have zero inner measure.

More generally, if a fixed set $B$ of positive measure is under consideration, a subset $S$ of $B$ will be called $s$-non-mble. if both $S$ and its complement relative to $B$ have zero inner measure. This implies $m_{*}(S)=0, m^{*}(S)=m(B)$ but is implied by these conditions only if $m(B)$ is finite.

Our object, in part, is to show that if $B$ is either the set of all real numbers or any half-open finite interval, then for every infinite cardinal $k \leqq C$ (the power of the continuum), $B$ can be partitioned into $k$ disjoint subsets which are $s$-non-mble. and are mutually congruent under translation (modulo the length of $B$ in the case that $B$ is a finite interval). Sierpinski and Lusin ${ }^{1}$ have partitioned $B$ into continuum many disjoint $s$-non-mble. subsets but they are not constructed to be congruent under translation. Other well known constructions do partition $B$ into a countable infinity of mutually congruent non-measurable subsets, but the subsets are not constructed to be saturated non-measurable. ${ }^{2}$

Presented to the Society, September 10, 1948 under the title Hamel's basis and non-measurable sets; received by the editors December 5, 1949 and, in revised form, March 20, 1950.

${ }^{1}$ C. R. Acad. Sci. Paris vol. 165 (1917) pp. 422-424.

2 See Hahn and Rosenthal, Set functions, University of New Mexico Press, 1948, pp. 102-104. The construction of $\$ 8.3 .3$ on p. 102 (as will be shown below) does give an $s$-non-mble. set but this is not proved there. 
2. Various authors have shown that if the axiom of choice is assumed, there are discontinuous solutions of the equation $f(x+y)$ $=f(x)+f(y)$ and that these solutions all have certain pathological properties. They are unbounded and non-measurable on every interval, indeed for all numbers $a, b$ the sets $E[x: f(x)<a]$ and $E[x: f(x)>b]$ have zero inner measure, which implies that they are $s$-non-mble. A deeper theorem of Ostrowski ${ }^{3}$ shows that if $a<b$, then the set $E[x: f(x)<a$ or $f(x)>b]$ has zero inner measure, which implies that the set $E[x: a \leqq f(x)<b]$ is $s$-non-mble.

Now suppose that $f(x)$ is such a discontinuous solution and that $d$ is any number greater than 0 for which $f\left(x_{0}\right)=d$ for some $x_{0}$. Let $S_{n}$ denote the set $E[x: n d \leqq f(x)<d(n+1)]$. Then the $S_{n}, n=0, \pm 1$, $\pm 2, \cdots$, are a partition of the set of all real numbers into a countable infinity of disjoint $s$-non-mble. sets which are congruent under translation since $S_{n+1}=x_{0}+S_{n}$. If $B$ is the interval $a \leqq x<b$ and $f(x)$ has the property $f(b-a)=0$, then the $B S_{n}$ are a partition of this kind of $B$ (modulo the length of $B$ ).

3. The theorem of Ostrowski does not state that the set $E(c)$ $=E[x: f(x)=c]$ is $s$-non-mble. and this is not true in general. However, we have the following theorem.

THEOREM. If $f(x)$ is a discontinuous solution of $f(x+y)=f(x)+f(y)$ which assumes only a countable number of distinct values, $c_{1}, c_{2}, \cdots$, $c_{n}, \cdots$, and $E_{n}$ denotes $E\left(c_{n}\right)$, then the $E_{n}$ are $a$ partition of the set of all real numbers into disjoint, s-non-mble. sets which are congruent under translation. If $B$ is an interval $a \leqq x<b$ and $f(x)$ has the additional property $f(b-a)=0$, then the $B E_{n}$ are such a partition of $B$ (modulo the length of $B)$.

Proof. If $x_{n}$ is an $x$ for which $f(x)=c_{n}$, then $E_{m}=\left(x_{m}-x_{n}\right)+E_{n}$ so that the $E_{n}$ are congruent under translation. They are obviously disjoint, and their set-union is the set of all real numbers. It remains to show that they are $s$-non-mble.

If $f(x) \neq 0$ for all $x \neq 0$, then $E(0)$ would consist of one number, namely 0 ; each $E_{n}$ would consist of one number since it is a translation of $E(0)$, which would give the false result that the set of all real numbers is countable. This shows that $f(\delta)=0$ for some $\delta>0$ and hence for all $r \delta, r$ rational but arbitrary.

Let $E_{n}(\alpha, \beta)$ denote the set of $x$ in $E_{n}$ for which $\alpha \leqq x<\beta$. Now $E_{n}(0, \delta)$ is not a null set, for if it were so we could deduce that

${ }^{3}$ Alexander Ostrowski, Über die Funktionalgleichung der Exponentialfunktion und verwandte Funktionalgleichungen, Jber. Deutschen Math. Verein. vol. 38 (1929.) 
$E_{n}(m \delta,(m+1) \delta)$ (by translation) then $E_{n}$ by set-union, and finally the set of all real numbers (by set-union of all $E_{n}$ ) were null sets. Hence $m^{*}\left(E_{n}(0, \delta)\right)=h \delta$ for some $0<h \leqq 1$ and some fixed $n$. It follows that $m^{*} E_{n}(r \delta, s \delta)=h(s-r) \delta$ for all rational $r, s$ with $r<s$; this is clear for $r=0$ and $s$ equal to a positive integer, or to the reciprocal of a positive integer, or, finally, to any positive rational; by translation the statement then follows for all rational $r, s$. Now, from the continuity of $m^{*}$ it follows that $m^{*}\left(E_{n} I\right)=h$ (length of $I$ ) for all intervals $I$.

Suppose, if possible, that $h<1$. Then the complement of $E_{n}$ has positive inner measure and hence there is an interval $I$ such that $m_{*}\left(I-E_{n} I\right)>(1-h) m(I)$. This gives $m^{*}\left(E_{n} I\right)<h m(I)$, a contradiction. Thus $h=1,{ }^{4}$ and $m^{*}\left(E_{n} I\right)=m(I)$ for all intervals $I$. This implies that $E_{n}$ is $s$-non-mble.

4. Let $e_{1}, e_{2}, \cdots, e_{\alpha}, \cdots$ be any Hamel's basis so that each $x$ has a unique expression $x=\sum_{\alpha} x_{\alpha} e_{\alpha}$ where the $x_{\alpha}$ are all rational and at most a finite number of them differ from zero. Let $S_{r}$ be the set of $x$ for which $x_{1}$ equals a given rational $r$. Then if $f(x)$ is defined to be $x_{1}$ for every $x$, the values of $f(x)$ will be countable and $\$ 3$ above shows that the $S_{r}$ are all $s$-non-mble. and a partition (into a countable infinity of subsets) of the desired kind of the set of all real numbers (for a finite interval $B$ it suffices to choose $e_{2}$ equal to the length of $B)$. This does not require the theorem of Ostrowski or any other theorem on the pathology of discontinuous solutions except as proved in $\$ 3$ above.

5. If $e_{1}, e_{2}, \cdots, e_{\alpha}, \cdots$ is any Hamel's basis, then any set of conditions on the $x_{\alpha}$ involving at most a countable number of $\alpha$ will give a set of $x$ which is either empty, or the set of all real numbers, or $s$-non-mble. For let $f(x)$ be defined to be a solution of $f(x+y)$ $=f(x)+f(y)$ with $f\left(e_{\alpha}\right)=e_{\alpha}$ if the value of $x_{\alpha}$ is involved in the given conditions, and 0 otherwise. Then each set of precise restrictions on the $x_{\alpha}$ involved in the original conditions, $x_{\alpha}=r_{\alpha}$, for arbitrary rationals $r_{\alpha}$, defines an $s$-non-mble. set. The conclusion follows.

In particular, the conditions $x_{n}=0$ for $n=1,2, \cdots$ gives an $s$-nonmble. set as do the conditions $x_{n}>0$ for $n=1,2, \cdots$. It follows that if the set of all real numbers be considered as a group $G$ with the rational numbers as multipliers (operators), then a proper subgroup $G_{1}$ which admits the rationals as operators is $s$-non-mble. if the number of cosets of $G_{1}$ with respect to $G$ is countable.

- In this connection, see Jacobsthal and Knopp, Sitzungsberichte der Berliner Mathematischen Gesellschaft vol. 14 (1915) p. 121. 
6. Let $\Omega$ be the smallest ordinal number with continuum many predecessors and let the collection of all perfect sets be arranged as a sequence $P_{\alpha}, 1 \leqq \alpha<\Omega .{ }^{1}$ For each $1 \leqq \alpha<\Omega$ define $a_{\alpha}, b_{\alpha}$ by induction so that $a_{\alpha}, b_{\alpha}$ are elements of $P_{\alpha}$ and the $a_{\beta}, b_{\beta}$ with $\beta \leqq \alpha$ are linearly independent with respect to rational numbers as coefficients; if an interval $B: a \leqq x<b$ is under consideration, define $a_{0}=b-a$. Such $a_{\alpha}, b_{\alpha}$ exists since for each $\alpha, P_{\alpha}$ has continuum elements whereas there are less than continuum rational-linear combinations of the $a_{\beta}, b_{\beta}$ with $\beta<\alpha$. Then there exists a Hamel's basis containing all $a_{\alpha}$ and all $b_{\alpha}$ as members. If $k$ is any infinite cardinal less than or equal to $C$, there is a subset $H_{1}$ of some of the $b_{\alpha}$ with cardinal $k$. Let $S$ consist of all linear combinations of elements of $H-H_{1}$, using rational numbers as coefficients, and let $y$ be an arbitrary linear combination of elements of $H_{1}$ with rational coefficients. The sets $S+y$ form a decomposition of the whole line into $k$ disjoint, congruent (under translation; modulo length of $B$ if an interval $B$ is under consideration) subsets. Since each $S+y$ has points in every perfect set ( $S$ contains all $a_{\alpha}$ ), it follows that the $S+y$ are saturated non-mble.

QUEEN'S UNIVERSITY 